\title{
SPECTRAL PROBLEMS IN SPACES OF CONSTANT CURVATURE
}

\author{
RAFAEL D. BENGURIA \\ Departamento de Física, P. Universidad Católica de Chile, \\ Casilla 306, Santiago 22, CHILE \\ E-mail: rbenguri@fis.puc.cl
}

\begin{abstract}
Here, recent spectral properties of some linear and nonlinear problems in spaces of constant curvature are reviewed. In particular, isoperimetric inequalities for low eigenvalues of the Laplace-Beltrami operator with Dirichlet and Neumann boundary conditions on smooth bounded domains on both $\mathbb{S}^{n}$ and $\mathbb{H}^{n}$ are considered. Also, I consider the BrézisNirenberg problem in those spaces.

Keywords: Eigenvalues of the Laplacian; Spaces of Constant Curvature; Brézis-Nirenberg Problem
\end{abstract}

\section{Introduction}

During the last decade, there has been an increasing interest in the properties of several spectral problems in spaces of constant curvature, in particular in problems defined on the $n$-dimensional sphere and also on the hyperbolic space. In general, most properties of the analog problems defined on the Euclidean Space, do hold in the hyperbolic case, and even in the case of $\mathbb{S}^{n}$ as long as the domains are restricted to live on a hemisphere. The situation changes, however, when the domains are allowed to extend beyond a hemisphere of $\mathbb{S}^{n}$. Here I only address, as examples, two completely different situations. The first one being the isoperimetric inequalities for the low lying Dirichlet and Neumann eigenvalues. The second being the analog of the Brézis-Nirenberg problem on both $\mathbb{S}^{n}$ and $\mathbb{H}^{n}$. In the case of $\mathbb{S}^{n}$, when the domain extends beyond the hemisphere, many interesting new features do appear. In the last few years there have also been many articles on universal inequalities for eigenvalues of the Laplace-Beltrami operator on spaces of constant curvature, as well as spectral problems for higher order operators on those spaces (in particular the study of the analog of the clamped plate problem and many others), I will not review those results he $\equiv$ Section 2, I will review the isoperimetric problems for eigenvalues of the laplikctan and in Section 3, I will review the Brézis-Nirenberg problem in $\mathbb{S}^{n}$ and in $\mathbb{H}^{n}$. It is interesting to note (and that is the reason I am treating these two, certainly different problems, together) that for some of the same rea $\equiv$ the $\equiv$ of the typical isoperimetric inequalities for low eigenvalues of the lap.uctan fail $\overline{\bar{n} \pi}$ the case of geodesic caps that extend beyond the hemisphere in $\mathbb{S}^{n}$, one encounters a whole new class of unexpected solutions for the Brézis-Nirenberg problem on those domains. 


\section{Isoperimetric inequalities for eigenvalues of the Laplacian}

Consider the eigenvalue problem,

$$
-\Delta u=\lambda u, \quad \text { in } \Omega
$$

with $u=0$ in $\partial \Omega$, where $\Omega$ is a bounded domain in $\mathbb{R}^{n}$. It is well known that this problem has a sequence of values of $\lambda$ (the Dirichlet eigenvalues), i.e.,

$$
0<\lambda_{1}<\lambda_{2} \leq \lambda_{k} \overline{\overline{\bar{F}}},
$$

for which (1) has a nontrivial solution. Associated with each $\lambda_{k}$ there is an eigenfunction $u_{k} \in H_{0}^{1}(\Omega)$. Certainly, the eigenvalues $\lambda_{k}(\Omega)$ depend on the geometry of the domain. In 1877, Lord Rayleigh conjectured that among all domains of fixed area (in two dimensions) the circular domain has the lowest $\lambda_{1}$. Rayleigh's conjecture was proved independently by G. Faber (1923) and E. Krahn (1925). Moreover, Krahn extended this result to $n$ dimensions. This fact is known as the RayleighFaber-Krahn (RFK) inequality, which reads,

$$
\lambda_{1}(\Omega) \geq\left(\frac{C_{n}}{|\Omega|}\right)^{2 / n} j_{n / 2-1,1}^{2}=\lambda_{1}\left(\Omega^{*}\right),
$$

where $j_{m, 1}$ denotes the first positive zero of the Bessel function $J_{m}$ and $C_{n}$ is the volume of the unit sphere in $n$ dimensions. Here, $\Omega^{*}$ denotes a ball of the same volume of $\Omega$. Equality is obtained in (2) if and only if $\Omega$ is a ball. There are many different proofs of the RFK inequality. Perhaps the simplest is based on the use of the variational characterization of $\lambda_{1}$ together with properties of symmetric decreasing rearrangements of functions. The RFK inequality has been extended to many other situations. In particular to the Dirichlet problem for domains on $\mathbb{S}^{n}$ by E. Sperner [1], and d $\equiv$ ns on $\mathbb{H}^{n}$, by I. Cha $\equiv$ ]. In 1992, A. Melas [3] proved the stability of the RKK inequality in the euclikean space. Stability here refers to the fact that if for a bounded, convex domain in $\mathbb{R}^{n}, \lambda_{1}(\Omega)|\Omega|$ differs little from the value that this quantity assumes for the ball, then the domain is approximately a ball (Melas also proved the stability of the PPW inequality that I discuss below). The stability for the analog of the RFK inequality for domains on on $\mathbb{S}^{n}$ was proved by A. Avila [4].

Consider now the corresponding Neumann problem for a bounded smooth domain, $\Omega \subset \mathbb{R}^{n}, n \geq 2$. That is,

$$
-\Delta u=\mu u, \quad \text { in } \Omega,
$$

and $\partial u / \partial n=0$ in $\partial \Omega$. Then, there is a sequence of values, $0=\mu_{0}(\Omega)<\mu_{1}(\Omega) \leq$ $\mu_{2}(\Omega) \ldots$, for which there is a nontrivial solution of the Neumann problem. In 1954, G. Szegö proved the isoperimetric inequality (for the case $n=2$ ):

$$
\mu_{1}(\Omega) \leq \mu_{1}\left(\Omega^{*}\right)=\frac{\pi p_{1,1}^{2}}{A}
$$

where $p_{1,1}=1.8412 \ldots$ is the first positive zero of the derivative of $J_{1}(t)$. The analogous result for $n \geq 2$ was proven by $\mathrm{H}$. Weinberger in 1956 . The analog of the Szegö-Weinberger inequality $\mathbb{S}^{n}$ was proven by Mark Ashbaugh and myself in 1995, [5]. However, our proof only works for domains that are contained in a 
hemisphere. Whether the corresponding result holds for domains that extend beyond a hemisphere, is still an open problem. In the case of the hyperbolic space, there are technical difficulties, but essentially the original proof of Weinberger (in the Euclidean case) can be carried through for any bounded domain in $\mathbb{H}^{n}[2,5]$.

In this context, the next simplest inequality that one can consider concerns the ratio between the first two Dirichlet eigenvalues. In 1955, L. Payne, G.Pólya and $\mathrm{H}$. Weinberger [PPW] proved the universal inequality $\lambda_{2}(\Omega) / \lambda_{1}(\Omega) \leq 3$ for bounded, smooth domains in $\mathbb{R}^{2}$. The PPW inequality in $n$ dimensions reads, $\lambda_{2}(\Omega) / \lambda_{1}(\Omega) \leq(1+4 / n)$. There is a vast literature on universal inequalities for eigenvalues of the Laplacian (i.e., inequalities between eigenvalues which are independent of the domain), including recent works of H.-C. Yang, and collaborators, Harrell and Stubbe, Harrell and Hermi, Ashbaugh and Hermi, Levitin and Parnovski. At the same time, PPW conjectured the bound,

$$
\frac{\lambda_{2}(\Omega)}{\lambda_{1}(\Omega)} \leq \frac{\lambda_{2}\left(\Omega^{*}\right)}{\lambda_{1}\left(\Omega^{*}\right)}=\frac{j_{1,1}^{2}}{j_{0,1}^{2}} \approx 2,539, \quad \text { for all } \Omega \subset \mathbb{R}^{2}, \text { bounded } .
$$

The PPW conjecture was proven by Mark Ashbaugh and myself [6-8]. The proof of the PPW inequality (5) is done in five steps: i) The first is to use a Rayleigh-Ritz variational estimate for the second Dirichlet eigenvalue (the so called gap formula); ii) in the second step one exploits the degeneracy of the second eigenvalue (for the conjectured maximizing domain), and then averages, using a Center of Mass argument to insure the necessary orthogonality; more technically, one uses a Brower Fixed Point Theorem at this stage; iii) in the third step, one guesses the right variational trial function (the guess is obviously based on the properties of the maximizing domain). Then, one has to prove monotonicity properties for functions associated to the trial function; iv) then one uses symmetrization (symmetric decreasing rearrangements), and, finally, v) a comparison theorem due to G. Chiti.

The analogous result for domains in $\mathbb{S}^{n}$ was proved in [9], but, again, only for domains contained in a hemisphere. It is still an open problem to determine whether a PPW inequality, like (5) is true for domains in $\mathbb{S}^{n}$ extending beyond a hemis $\equiv$. The main reason (but not the only one) that our proof fails for domain that extends beyond a hemisphere is that we cannot prove the monotonicity properties associated to the trial functions (i.e., the main part of step iii) in the proof of PPW). This failure relies on the fact that the Laplace-Beltrami operator (in geodesic ccordinates) acting on radial functions has a term proportional to the radial derivative and $\mathrm{t} \equiv$ efficient changes sign when going beyond the hemi $\equiv$ re (see equation (9) below $\bar{r}$ n the contrary, for both the Euclidean and the Hyperbolic cases the corresponding coefficient is of one sign.

Concerning the hyperbolic space, $\mathrm{t} \rightleftharpoons$ prresponding result was proven by $\mathrm{H}$. Linde and myself [10]. In the case of $\mathbb{H}^{n}$ case one cannot hope to prove that $\lambda_{2} / \lambda_{1}(\Omega)$ is maximized by geodesic balls (this is in fact not true in general). The point here is that the ratio $\lambda_{2}(B) / \lambda_{1}(B)$, where $B$ is a geodesic ball, is not a decreasing function of the radius of the ball, as needed to complete the analog of the Chiti's comparison argument (step 5 in the proof of the eucli $\equiv$ case). Notice, that this ratio is independent of the radius of the ball in the euclidean case, and it has the right monotonicity property for domains in $\mathbb{S}^{n}$. Thus, in the $\mathbb{H}^{n}$ case what one proves is that 
among all domains having a fixed $\lambda_{1}(\Omega)$ eigenvalue, it is the geodesic ball the one that maximizes $\lambda_{2}$. For some recent detailed reviews on isoperimetric inequalities for eigenvalues of the laplacian, see, e.g., [11, 12].

\section{Brézis-Nirenberg problem in $\mathbb{S}^{n}$ and $\mathbb{H}^{n}$}

Consider the boundary value problem

$$
-\Delta u=\lambda u+u^{5},
$$

in $\Omega \subset R^{3}$, bounded, with Dirichlet boundary conditions. Brézis and Nirenberg [13] considered the problem of determining the range of values of the parameter $\lambda$ for which the above problem has a positive solution, $u>0$ in $\Omega$. If the domain $\Omega$ is a ball (of radius $R$, say), that range is precisely given by

$$
\frac{\lambda_{1}}{4}<\lambda<\lambda_{1}
$$

where $\lambda_{1}=\pi^{2} / R^{2}$ is the first Dirichlet eigenvalue of the ball. For general domains, there exists a value $0<\lambda_{1}^{*}(\Omega)<\lambda_{1}(\Omega)$, such that for $\lambda \in\left(\lambda_{1}^{*}, \lambda_{1}\right)$ the above problem has a positive solution. The proof of the Brézis-Nirenberg result goes in two steps: i) Nonexistence: For $\lambda \geq \lambda_{1}$, just multiply (6) by the first Dirichlet eigenfunction of the domain, integrate in $\Omega$ and you are done. Moreover, when the domain is a ball, use a refinement of the Rellich-Pohozaev identity in order to show that there are no positive solutions for $\lambda \leq \lambda_{1} / 4$.

ii) Existence: In order to show existence in the interval $\left(\lambda_{1} / 4, \lambda_{1}\right)$ (for the case of the ball), one uses the Brézis-Lieb compactness argument [14].

The corresponding problem,

$$
-\Delta_{\mathbb{S}^{3}} u=\lambda u+u^{5}
$$

for $u>0$ on geodesic balls $D^{\prime}\left(\theta^{*}\right)$ on $\mathbb{S}^{3}$ with Dirichlet boundary conditions was considered in [15]. Here, without loss of generality the geodesic ball is centered at the north pole, and $\theta^{*}$ is the azimuthal angle of the boundary of $D^{\prime}$. Using a moving plane method (more precisely a moving spheres method) introduced by Padilla [16] one can prove that th $\equiv$ itive solutions of (8) (when $\theta^{*} \leq \pi / 2$, i.e., when $D^{\prime}$ lies inside a hemisphere) arz radially symmetric, i.e., they only depend on the azimuthal angle $\theta$. In geodesic coordinates, for radial functions,

$$
\Delta_{\mathbb{S}^{3}} u=u^{\prime \prime}(\theta)+2 \cot \theta u^{\prime}(\theta)
$$

For domains lying inside a hemisphere the situation is similar to the euclydean case. One has the following result:

Theorem 3.1 ( [15]). If $\lambda>-3 / 4$, there is a positive solution to (8), if and only if,

$$
\lambda_{1}^{*}=\frac{\pi^{2}-4 \theta^{* 2}}{4 \theta^{*^{2}}}<\lambda<\frac{\pi^{2}-\theta^{* 2}}{\theta^{*^{2}}} .
$$

If $\lambda \leq-3 / 4$, and $\theta^{*} \in(0, \pi / 2]$ there are no positive solutions to (8). 
As in the Euclidean case, the existence part relies on the Brézis-Lieb compactness result. The nonexistence part relies on a refined Rellich-Pohozaev argument. The picture for the geodesic balls contained in a hemisphere is not surprising. It is to be expected, after the results of Bandle and Peletier on best critical constants for the Sobolev embeddings in $\mathbb{S}^{3}$, in the case $\lambda=0$ [17].

On the other hand, once we exceed the hemisphere, interesting new solutions start appearing. Originally, we proved the following result

Theorem 3.2 ( $[\mathbf{1 5}])$. Given any $\theta^{*} \in(\pi / 2, \pi)$, there exists at least two non constant solutions of the Brézis-Nirenberg problem on $D^{\prime} \subset \mathbb{S}^{3}$, as long as $\lambda$ is sufficiently large (and negative).

Later, Bandle and Stingelin [18] numerically found a double sequence of new positive solutions for domains exceeding the hemisphere. Then, independently, Chen and Wei [19], Brézis and Peletier [20], and Bandle and Wei [21, 22] completely classified all the positive solutions to this problem. In particular one has,

Theorem 3.3 ( [20]). Given any $\theta^{*} \in(\pi / 2, \pi)$, there exists a constant $A_{k}>0$ such that for $\lambda<-A_{k}$, the Brézis-Nirenberg problem has at least $2 k$ solutions, such that at the North-Pole, $u(0) \in\left(0,|\lambda|^{1 / 4}\right)$.

While in the $\mathbb{S}^{3}$ case there is this rich set of solutions once we exceed a hemisphere, in the hyperbolic case the situation is completely analogous to the one for the euclidean space. The analogous problem for geodesic balls in the hyperbolic space $\mathbb{H}^{3}$ (in fact for $\mathbb{H}^{n}$, with $n \geq 3$ ) was considered in the Ph.D. Thesis of Silke Stapelkamp [23]. She considers the following realization of $\mathbb{H}^{3}$ (as the positive branch of an hyperboloid embedded in $\left.\mathbb{R}^{4}\right)$ :

$$
\mathbb{H}^{3}=\left\{x \in \mathbb{R}^{4} \mid x_{1}^{2}+x_{2}^{2}+x_{3}^{2}-x_{4}^{2}=1, x_{4}>0\right\}
$$

and, moreov $\equiv$ e considers the stereographic projection of $\mathbb{H}^{3}$ into $\mathbb{R}^{3}$ (in fact into the euclidean apsce defined as $\left.x_{4}=0\right)$, from the point $(0,0,0,-1)$ in $\mathbb{R}^{4}$. Then, among many other results (including results for geodesic balls in $\mathbb{H}^{n}$, with $n \geq 4$ ), she proved the following theorem for geodesic balls in $\mathbb{H}^{3}$.

Theorem 3.4 ( $[23])$. Let $D^{\prime}$ be a geodesic ball in $\mathbb{H}^{3}$, with center at $(0,0,0,1)$. Let $D=B(0, R), 0<R<1$ be the stereographic projection of $D^{\prime}$ into $\mathbb{R}^{3}$. Let,

$$
\lambda^{*}=1+\frac{\pi^{2}}{16\left(\tanh ^{-1}(R)\right)^{2}} .
$$

Then,

i) If $\lambda \leq \lambda^{*}$, or $\lambda \geq \lambda_{1}$, the Brézis-Nirenberg problem on $D^{\prime}$ has only trivial solutions.

ii) Moreover, if $\lambda \in\left(\lambda^{*}, \lambda_{1}\right)$, there is a nontrivial positive solution to the BrézisNirenberg problem on $D^{\prime}$.

Remark: Here, $\lambda_{1}$ denotes the first Dirichlet eigenvalue of the Laplace-Beltrami operator on $D^{\prime} \subset \mathbb{H}^{3}$. It is clear from (10) that $\lambda^{*} \rightarrow 1$ as $R \rightarrow 1$. Moreover, one can check also that $\lambda_{1}(R) \rightarrow 1$ as $R \rightarrow 1$. The proof of this theorem follows the same steps as in the case of $\mathbb{R}^{3}$ and $\mathbb{S}^{3}$. In particular, it uses the fact that the positive 
solutions of the Brézis-Nirenberg equation on $D^{\prime} \subset \mathbb{H}^{3}$ are radial, which is proved using a generalization of the moving planes method. For higher dimensions, if $D^{\prime}$ is now any bounded domain in $\mathbb{H}^{n}, n \geq 4$, the Brézis-Nirenberg problem does not have nontrivial solutions if $\lambda \geq \lambda_{1}$, nor does it if it is star shaped, and $\lambda \leq n(n-2) / 4$. On the other hand, there exists a nontrivial solution if $\lambda \in\left(n(n-2) / 4, \lambda_{1}\right)$. The proof of existence relies on a concentration-compactness argument, while the nonexistence result for star shaped domains, when $\lambda<n(n-2) / 4$, relies on a Rellich-Pohozaev argument.

\section{Acknowledgment}

This article is dedicated to Ari Laptev for his birthday celebration. This work has been supported by the ICM (CHILE) project P07-027-F. I would like to thank the organizers of QMath11 for their kind invitation and for their hospitality in Hradec Kralove.

\section{References}

[1] E. Sperner, Math. Z. 134, 317 (1973).

[2] I. Chavel, Lowest eigenvalue inequalities, in Geometry of the Laplace operator, ed. Osserman, R. and Weinstein, A., Proc. Symposia Pure Mathematics, Vol. 361994.

[3] A. Melas, J. Diff. Geometry 36, 19 (1992).

[4] A. Avila, J. Math. Anal. Appl. 267, $760 \equiv$ 三).

[5] M. Ashbaugh and R. Benguria, Journal Of The London Mathematical Society-Second Series 52, 402(1995).

[6] M. Ashbaugh and R. Benguria, Bulletin of The American Mathematical Society 25, 19 (1991).

[7] M. Ashbaugh and R. Benguria, Annals Of Mather $\equiv$ 135, 601( 1992).

[8] M. Ashbaugh and R. Benguria, Communications In Mathematical Physics 147, 181 (1992).

[9] M. Ashbaugh and R. Benguria, Transactions of The American Mathematical Society 353, 1055 (2000).

[10] R. D. Benguria and H. Linde, Duke Mathematical Journal 140, 245 (2007).

[11] M. S. Ashbaugh and R. D. Benguria $\bar{\equiv}$ perimetric Inequalities For I-Sigenval飞es Of The Laplacian, in Spectral Theory An Mathematical Physics: A Festschrift In Honor Of Barry Simon's 60th Birthday - Quantum Field Theory, Statistical Mechanics, And Nonrelativistic Quantum Systems, ed. Gesztesy, F, Deift, P., Galvez, C., Perry, P. and Schlag, W, Proceedings Of Symposia In Pure Mathematics, Vol. 76(Part 1)2007.

[12] R. D. Benguria and H. Linde, Isoperimetric Inequalities For Eigenvalues Of The

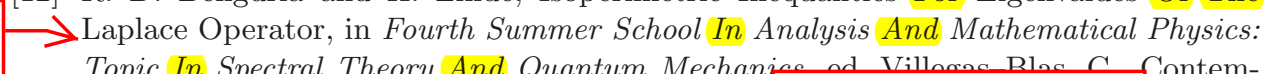

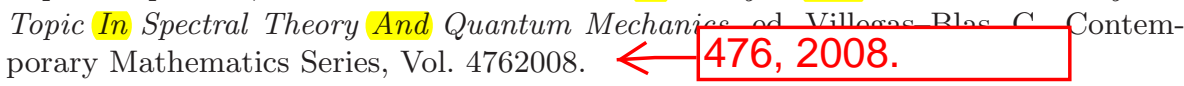

[13] H. Brézis and L. Nirenberg, Comm. Pure Appl. Math. 36, 437 (1983).

[14] H. Brézis and E. Lieb, Proc. Amer. Math. Soc. 88, 486 (1983).

[15] C. Bandle and R. Benguria, J. Differential Equations 178, 59 (2002).

[16] P. Padilla, Applicable Analysis 64, 153 (1997).

[17] C. Bandle and L. Peletier, Math. Annalen 313, 83 (1999).

[18] C. Bandle and S. Stingelin, Progess in Nonlinear Differential Equations and their Applications 63, 13 (2005).

[19] W. Y. Chen and J. C. Wei, Comptes Rendus Mathematique 341, 153 (2005). 
[20] H. Brézis and L. Peletier, J. D'Analyse Mathematique 98, 279 (2006).

[21] C. Bandle and J. Wei, Journal D'Analyse Mathematique 102, p. 2007 (181-208).

[22] C. Bandle and J. Wei, Commun. Partial Differential Equations 33, 613 (2008).

[23] S. Stapelkamp, The Brézis-Nirenberg problem on $\mathbb{H}^{n}, \mathrm{PhD}$ thesis, University Of Basel, (Basel, Switzerland, 2002). 